\title{
Denervação Renal com Cateter de Ablação por Radiofrequência de Ponta Irrigada em Hipertensos Resistentes
}

\author{
Rodolfo Staico, Luciana Armaganijan, Dalmo Moreira, Marcio Sousa, Flavio Borelli, Celso Amodeo, \\ Jônatas Melo Neto, J. Eduardo Sousa, Amanda Sousa, Alexandre Abizaid
}

\section{RESUMO}

Introdução: Resultados de 3 anos do Symplicity HTN-1 mostraram superioridade da denervação simpática renal percutânea comparada à terapia medicamentosa em hipertensos resistentes. Entretanto, os desfechos de eficácia da denervação simpática renal percutânea no Symplicity HTN-3, primeiro ensaio controlado com procedimento simulado, não foram alcançados. Nosso objetivo foi relatar os resultados de segurança e de eficácia da denervação simpática renal percutânea utilizando o cateter de ablação por radiofrequência de ponta irrigada em hipertensos resistentes. Métodos: Vinte e um pacientes foram submetidos ao procedimento desde julho 2012, dentre eles nove com seguimento $\geq 12$ meses, os quais foram incluídos nesta análise. O desfecho primário foi a segurança, avaliada por eventos adversos peri-procedimento, função renal e anormalidade vascular renal aos 6 meses. Os desfechos secundários incluíram mudanças na pressão arterial obtida no consultório e no número de anti-hipertensivos aos 12 meses. Resultados: A média de idades foi 48,8 \pm 11,7 anos. No primeiro caso, houve dissecção de artéria renal causada por trauma da bainha. Nenhum caso de trombose, infarto renal ou óbito foi observado. Não se constatou elevação dos níveis séricos de creatinina durante o seguimento. Aos 6 meses, diagnosticou-se um caso de estenose significativa de artéria renal, sem repercussão clínica. A redução média da pressão arterial no consultório foi de 41,1 $\pm 33,2 / 18,6 \pm$ $15,2 \mathrm{mmHg}$ ( $p=0,04$ para pressão arterial sistólica e $p=0,08$ para pressão arterial diastólica) e houve redução de 2,0 \pm 2,3 no número de anti-hipertensivos aos 12 meses $(p=0,03)$. Conclusões: A denervação simpática renal percutânea, utilizando o cateter de ablação por radiofrequência de ponta irrigada, mostrou ser factível, segura e eficaz.

DESCRITORES: Hipertensão. Resistência a medicamentos. Ablação por cateter.

\section{ABSTRACT \\ Renal Denervation Using an Irrigated \\ Radiofrequency Ablation Catheter in Patients with Resistant Hypertension}

Background: The 3-year results of the Symplicity HTN-1 trial have shown the superiority of renal sympathetic denervation over drug treatment in patients with resistant hypertension. However, the efficacy of renal sympathetic denervation was not confirmed by Symplicity HTN-3, the first sham-controlled study. Our objective was to report the safety and efficacy of renal sympathetic denervation using the irrigated radiofrequency ablation catheter in patients with resistant hypertension. Methods: Twenty-one patients were submitted to the procedure since July 2012, nine of them had a follow-up $\geq 12$ months and were included in this analysis. The primary endpoint was safety, evaluated by periprocedural adverse events, renal function and renal vascular abnormalities at 6 months. Secondary endpoints included changes in blood pressure obtained in office and in the number of anti-hypertensive drugs at 12 months. Results: Mean age was $48.8 \pm 11.7$ years. In the first case, there was a renal artery dissection caused by the vascular sheath. There were no cases of thrombosis, renal infarction or death. Serum creatinine levels did not increase during the follow-up period. At 6 months, one case of significant renal stenosis without clinical impact was diagnosed. The average reduction in office blood pressure was $41.1 \pm$ $33.2 / 18.6 \pm 15.2 \mathrm{mmHg}$ ( $p=0.04$ for systolic blood pressure and $p=0.08$ for diastolic blood pressure) and there was a mean reduction of $2.0 \pm 2.3$ in the number of anti-hypertensive drugs at 12 months $(p=0.03)$. Conclusions: Renal sympathetic denervation using the irrigated radiofrequency ablation catheter proved to be feasible, safe and effective.

DESCRIPTORS: Hypertension. Drug resistance. Catheter ablation. 
C om o objetivo de promover a inibição ou o bloqueio da atividade do sistema nervoso simpático, denervação simpática renal (DSR) percutânea surgiu como perspectiva de tratamento adjunto para pacientes portadores de hipertensão arterial resistente. A comunidade médica tem acompanhado e se entusiasmado com os resultados da DSR desde a publicação de Schlaich et al.,' na qual foi relatada, pela primeira vez, a redução da pressão arterial (PA) em paciente hipertenso resistente (em uso de sete diferentes fármacos anti-hipertensivos) de 161/107 mmHg para $127 / 81 \mathrm{mmHg}$, após o procedimento. É notável o crescimento exponencial das pesquisas e do número de publicações referentes ao tema. ${ }^{2}$ Empresas de dispositivos médicos avaliaram a DSR como grande inovação e como terapia de sucesso para milhões de pacientes e, hoje, contabilizam-se mais de 60 companhias envolvidas com essa modalidade de tratamento. Ensaios como o Symplicity HTN-1 ${ }^{3}$ e Symplicity HTN- $2^{4}$ mostraram decréscimos significativos da PA, atestando a eficácia e a segurança da DSR. Três anos de seguimento do Symplicity HTN-1 revelaram a manutenção dos resultados em longo prazo, com diminuição da PA de 32/14 mmHg. ${ }^{5}$ Esses resultados inéditos superaram aqueles alcançados com a terapia medicamentosa e contribuíram para aumentar o entusiasmo com a nova técnica.

O Symplicity HTN-3, um estudo randomizado, cego e controlado com procedimento simulado (sham control), foi apresentado no congresso do American College of Cardiology e publicado on-line no New England Journal of Medicine no mesmo dia, em março de 2014. Bhatt et al. ${ }^{6}$ causaram polêmica com a apresentação dos resultados. Aos 6 meses, a redução relativa da PA sistólica (PAS) foi semelhante para ambos os grupos ( $p<0,001$ para as mudanças relativas aos níveis pressóricos pré-DSR, nos dois grupos), com uma diferença entre os grupos de apenas $-2,39 \mathrm{mmHg}$ a favor da DSR. Além disso, uma diferença pré-especificada na PAS aferida pela monitorização ambulatorial da PA (MAPA) de 24 horas de apenas $2 \mathrm{mmHg}$ não foi atingida. Assim, os desfechos primário e secundário de eficácia da DSR, no Symplicity HTN-3, não foram alcançados. Os resultados contrariaram a maior parte dos trabalhos publicados previamente, apesar de um estudo recente sugerir inferioridade da DSR quando comparada ao tratamento medicamentoso ajustado. ${ }^{7}$

À primeira vista, a explicação mais plausível para os resultados desfavoráveis do Symplicity HTN-3 foi a inclusão de um grupo controle com procedimento simulado. Em ensaios clínicos testando intervenções e dispositivos médicos, o controle com procedimento simulado é análogo ao da utilização de um placebo, em ensaios farmacêuticos. No entanto, por razões éticas, o controle com procedimento simulado é usualmente desencorajado. Tanto o Symplicity HTN-1 como o HTN-2 não tiveram grupo controle com procedimento simulado.
Por essa razão, o efeito placebo pode explicar toda ou a maior parte das diferenças de PA observadas nos primeiros dois ensaios. A falta de eficácia da DSR pode também ter sido causada por denervação incompleta ou ineficaz. Não há menção dos marcadores de eficácia da DSR, como, por exemplo, a microneurografia ou o spillover de norepinefrina, métodos que medem direta e indiretamente a atividade simpática, respectivamente. No entanto, o cateter de ablação utilizado no Symplicity HTN-3 foi o mesmo utilizado nos estudos Symplicity HTN-1 e HTN-2.

No Brasil, entretanto, não havia disponibilidade de cateteres dedicados para DSR até há pouco tempo. Mesmo assim, apesar de dois sistemas, o Symplicity $^{\circledR}$ (Medtronic ${ }^{\circledR}$, Minneapolis, Estados Unidos) e o EnligHTN $^{\circledR}$ (St Jude Medical ${ }^{\circledR}$, Minneapolis, Estados Unidos), já estarem aprovados pela Agência Nacional de Vigilância Sanitária (ANVISA) para uso clínico, eles ainda não são usados de rotina no nosso meio. Amparados nas potenciais vantagens do cateter irrigado nas ablações cardíacas, que causam lesões mais profundas com menor risco de formação de trombos na ponta do cateter, começamos a utilizar esse tipo de cateter para a DSR há quase 2 anos, baseados na hipótese de que cateteres com ponta irrigada podem ser benéficos nesse contexto, e objetivando avaliar a factibilidade e a segurança da DSR com cateter irrigado em pacientes com HAS resistente. Recentemente, publicamos nossos resultados dos dez primeiros pacientes submetidos ao procedimento, com seguimento de 6 meses. ${ }^{8}$ Nosso objetivo foi relatar os resultados de até 12 meses da DSR utilizando o cateter irrigado nessa população.

\section{MÉTODOS}

\section{Casuística}

Foram avaliados para inclusão no estudo pacientes com HAS resistente em acompanhamento regular no Ambulatório de Hipertensão Arterial e Nefrologia do Instituto Dante Pazzanese de Cardiologia. A definição de hipertensão resistente foi embasada no consenso da American Heart Association, ${ }^{9}$ de maneira que os pacientes deveriam apresentar PAS aferida em consultório $>140 \mathrm{mmHg}$, a despeito do uso de três ou mais fármacos anti-hipertensivos, ou PA controlada com $\geq 4$ anti-hipertensivos com ação sinérgica. O diagnóstico de HAS resistente deveria ter sido confirmado por, ao menos, uma MAPA ao longo do acompanhamento.

Todas as aferições de PA foram realizadas com esfigmomanômetro braquial automático e de acordo com as normas da VI Diretriz Brasileira de Hipertensão Arterial. ${ }^{10} \mathrm{~A}$ escolha do tratamento medicamentoso para o controle dos níveis tensionais ficou a critério do médico assistente, de acordo com o perfil de tolerância e as comorbidades associadas. A adesão ao tratamento foi encorajada em todas as consultas médicas. 
A má adesão ao tratamento medicamentoso foi excluída por meio de internação por pelo menos 5 dias antes da intervenção. O período de hospitalização se prestou, também, a completar e atualizar a investigação de causas secundárias para HAS.

Excluíram-se pacientes com taxa de filtração glomerular < $45 \mathrm{~mL} / \mathrm{min}$; hepatopatia grave; distúrbios de coagulação; insuficiência cardíaca classe funcional III ou IV (segundo classificação funcional da New York Heart Association - NYHA); disfunção ventricular grave (fração de ejeção - FE < 0,30); valvopatias moderadas ou graves; arritmias graves; infarto agudo do miocárdio ou acidente vascular encefálico nos últimos 6 meses; angina e/ou isquemia miocárdica não esclarecidas; anomalias de artéria renal (estenose $>50 \%$, presença de stent ou angioplastia prévia, rim único); diâmetro da artéria renal $<4 \mathrm{~mm}$; antecedentes cirúrgicos nos últimos 2 meses; hipersensibilidade ao meio de contraste; suspeita de gravidez; antecedentes de etilismo num período inferior a 1 ano; doença psíquica que comprometesse o acompanhamento; e expectativa de vida $<1$ ano.

O protocolo deste projeto de pesquisa, assim como o formulário de consentimento informado, foi devidamente aprovado pelo Comitê de Ética em Pesquisa do Instituto Dante Pazzanese de Cardiologia. Todos os pacientes assinaram o Termo de Consentimento Livre e Esclarecido.

\section{Desfechos clínicos}

O desfecho primário do estudo foi a segurança do procedimento, avaliada por meio da quantificação da taxa de eventos adversos peri-procedimento (complicações vasculares no sítio de punção e na artéria renal), análise da função renal (comparação entre o estado basal e 6 meses após a intervenção) e ocorrência de estenose/aneurisma na artéria renal aos 6 meses após a intervenção, avaliada pela arteriografia.

Os desfechos secundários constituíram as mudanças da PA aferida em consultório aos 12 meses de seguimento, bem como a análise do efeito do procedimento sobre o número de fármacos anti-hipertensivos nesse período.

\section{Descrição do procedimento}

Dois acessos vasculares foram obtidos, de acordo com método padronizado pela equipe, sendo um em artéria femoral direita e outro em artéria femoral esquerda. A artéria femoral esquerda foi utilizada para inserção de cateter com a finalidade de injetar meio de contraste, para realização da arteriografia renal. A artéria femoral direita foi utilizada para a inserção de uma bainha, através da qual o cateter de ablação Therapy Cool Point ${ }^{\circledR}$ (St Jude Medical ${ }^{\circledR}$, Minneapolis, Estados Unidos) ou Celsius Thermocool ${ }^{\circledR}$ (Biosense-Webster Inc ${ }^{\circledR}$, Diamond Bar, Estados Unidos) foi introduzido e posicionado na artéria renal. Após a obtenção do acesso vascular, heparina não fracionada foi administrada na dose de $100 \mathrm{U} / \mathrm{kg}$ para anticoagulação. Aortografia junto às artérias renais, seguida de cateterização e arteriografia renal seletiva, para avaliação da anatomia e do posicionamento do cateter de ablação na porção distal, foi realizada após a administração de nitroglicerina intravascular (200 mcg). Quatro a seis aplicações sequenciais de radiofrequência foram realizadas ao longo de ambas as artérias renais com a finalidade de promover a denervação renal. O cateter foi tracionado $5 \mathrm{~mm}$ e girado após cada aplicação, configurando uma disposição helicoidal das ablações (Figura 1).

Devido à intensa dor visceral gerada pela ablação, sedação/analgesia com narcóticos e opioides foi realizada em todos os casos. Ao final do procedimento, arteriografia renal de controle foi realizada para avaliação da integridade vascular, após administração de nitroglicerina intravascular (200 mcg).

\section{Avaliação pós-procedimento e seguimento clínico}

Os introdutores foram removidos quando o tempo de coagulação ativado atingiu valores < 200 seg. Compressão hemostática manual foi realizada por, pelo menos, 20 minutos, seguida de curativo compressivo. A deambulação foi permitida após 4 horas de repouso, na ausência de sangramento no sítio de punção. Após o procedimento, foi dada atenção à ocorrência de complicações vasculares no acesso femoral, como hematomas e pseudoaneurismas. As medicações foram ajustadas e os pacientes, orientados quanto à necessidade de acompanhamento periódico.

O primeiro retorno foi realizado $7 \pm 2$ dias após o procedimento e teve por finalidade avaliar complicações no sítio de punção, função renal, hemoglobina/ hematócrito e reajustar a dose dos anti-hipertensivos. Os retornos seguintes foram realizados em $30 \pm 7$, $90 \pm 10,180 \pm 10$ e $360 \pm 10$ dias. A aferição da PA foi a mesma descrita para a avaliação pré-procedimento. Exames laboratoriais foram solicitados em todas as visitas. Angiografia de artérias renais foi repetida aos 6 meses de seguimento.

\section{Análise estatística}

Variáveis contínuas foram expressas como média e desvio padrão, e comparadas utilizando-se o teste $t$ de Student; as variáveis categóricas foram apresentadas como frequências absolutas e relativas. Para todos os parâmetros, valores de $p \leq 0,05$ foram considerados estatisticamente significativos. Os dados foram analisados utilizando-se o programa Statistical Package for Social Sciences $^{\circledR}\left(\right.$ SPSS $\left.^{\circledR}\right)$, versão 16, software para Windows ${ }^{\circledR}$ (SPSS Inc ${ }^{\circledR}$, Chicago, Illinois, EUA). 

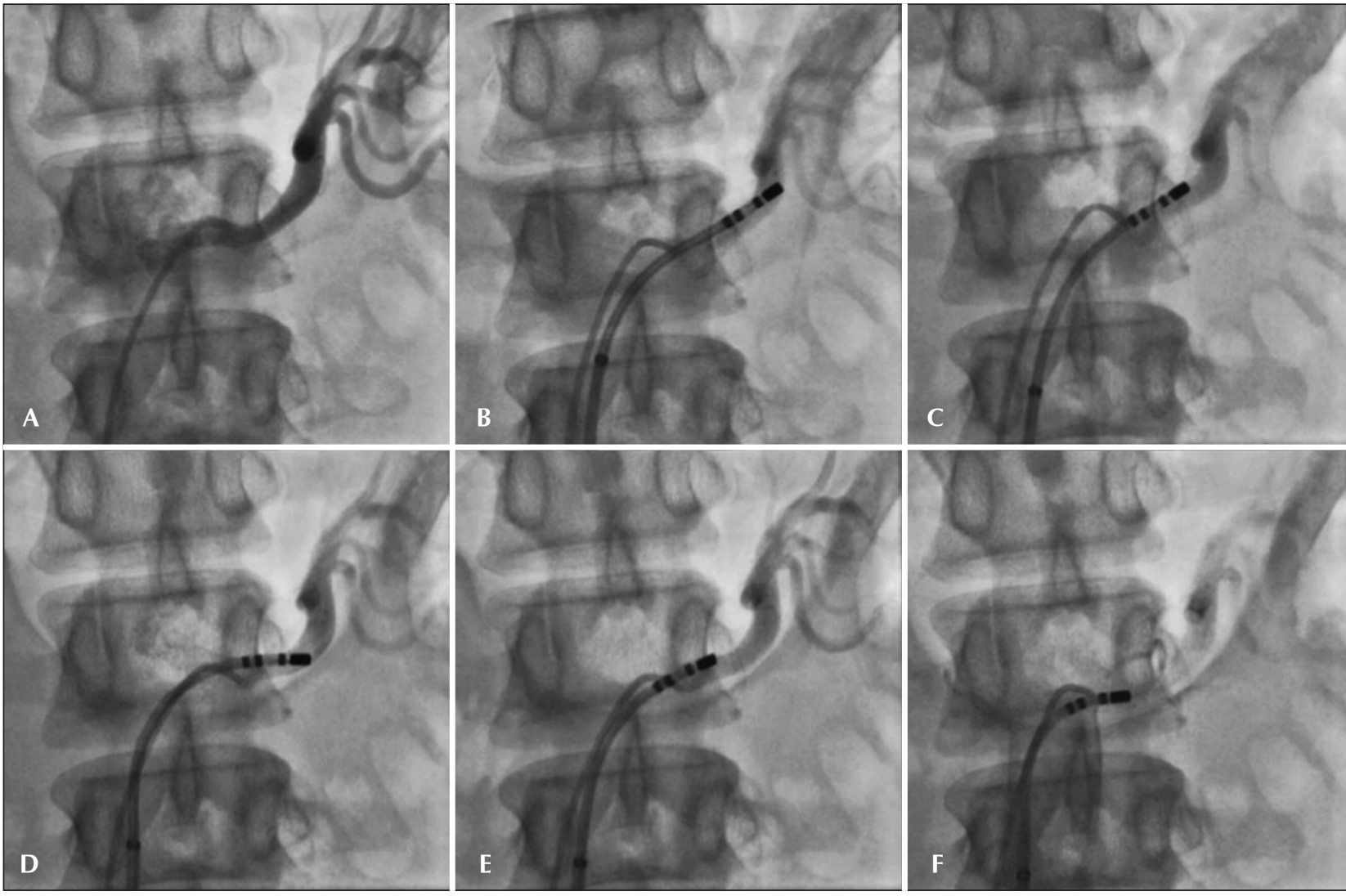

Figura 1. Lesões sequenciais de radiofrequência em artéria renal. (A) Angiografia seletiva de artéria renal esquerda; (B a F) aplicações sequenciais iniciadas no segmento distal; o cateter foi tracionado e rodado após cada aplicação para promover lesões sequenciais com configuração helicoidal.

\section{RESULTADOS}

\section{Características clínicas}

Vinte e um pacientes foram submetidos à DSR para tratamento adjunto da HAS resistente. Dentre eles, nove (oito mulheres) com seguimento $\geq 12$ meses foram incluídos na presente análise. A média de idade foi $48,8 \pm 11,7$ anos. O tempo médio do diagnóstico de HAS foi 18,1 $\pm 11,3$ anos. As médias de PAS e PA diastólica (PAD) medidas no consultório foram 202,8 \pm $32,5 \mathrm{mmHg}$ e $116,2 \pm 18,7 \mathrm{mmHg}$, respectivamente. O número médio de anti-hipertensivos antes do procedimento foi $7,7 \pm 1,3$. As características clínicas dos pacientes encontram-se resumidas na tabela 1 .

\section{Características angiográficas}

Os diâmetros médios das artérias renais direita e esquerda foram 5,0 $\pm 0,4 \mathrm{~mm}$ e 5,0 $\pm 0,7 \mathrm{~mm}$, respectivamente. As extensões médias das artérias renais direita e esquerda foram 33,7 $\pm 7,0 \mathrm{~mm}$ e $26,4 \pm 10,3 \mathrm{~mm}$, respectivamente.

Em um caso, observou-se a presença de uma artéria polar à esquerda e, em outro, notou-se a presença de três artérias polares, sendo duas à esquerda e uma à
TABELA 1

Características basais

\begin{tabular}{lc}
\hline Variável & $\mathbf{n}=\mathbf{9}$ \\
\hline Idade, anos & $48,8 \pm 11,7$ \\
Índice de massa corporal, $\mathrm{kg} / \mathrm{m}^{2}$ & $29,9 \pm 3,9$ \\
Diabetes mellitus, $\mathrm{n}(\%)$ & $3(33,3)$ \\
Dislipidemia, $\mathrm{n}(\%)$ & $7(77,8)$ \\
Tabagismo, $\mathrm{n}(\%)$ & 0 \\
Etilismo, $\mathrm{n}(\%)$ & 0 \\
Infarto do miocárdio prévio, $\mathrm{n}(\%)$ & 0 \\
Acidente vascular cerebral prévio, $\mathrm{n}(\%)$ & 0 \\
Doença vascular periférica, $\mathrm{n}(\%)$ & 0 \\
Antecedente familiar de HAS, $\mathrm{n}(\%)$ & $8(88,9)$ \\
Creatinina basal, mg/dL & $0,96 \pm 0,29$ \\
Tempo de diagnóstico da HAS, anos & $18,1 \pm 11,3$ \\
PAS/PAD aferidas no consultório, mmHg & $202,8 \pm 32,5 / 116,2 \pm 18,7$ \\
\hline HAS: hipertensão arterial sistêmica; PAS: pressão arterial sistólica; PAD: pressão \\
arterial diastólica.
\end{tabular}

direita. Duas pacientes apresentaram estenose discreta proximal em artéria renal (Tabela 2). 


\section{Características técnicas do procedimento}

Todos os procedimentos foram realizados por via transfemoral, conforme protocolo aqui descrito. Em média, foram utilizados $76,7 \pm 25,4 \mathrm{~mL}$ de meio de contraste durante 19,7 $\pm 6,2$ minutos de fluoroscopia. O número médio de aplicações de radiofrequência foi 5,2 $\pm 0,7$ na artéria renal direita e 4,3 $\pm 1,7$ na artéria renal esquerda (Tabela 2).

\section{Segurança do procedimento}

O procedimento foi realizado sem complicações em oito casos. No primeiro caso dessa série, foi detectada dissecção de artéria renal após a realização da ablação por trauma mecânico causado pela bainha. A mesma foi tratada com implante de stent, sem complicações subsequentes ou aumento no tempo de hospitalização. Aos 6 meses, arteriografia renal de controle revelou stent pérvio e sem hiperplasia significativa intra-stent.

Irregularidades parietais focais na artéria renal foram observadas em alguns casos imediatamente após a aplicação de radiofrequência, tendo sido atribuídas a espasmo e/ou edema. Nenhuma foi considerada limitante ao fluxo sanguíneo ao final do procedimento.

Não houve complicações relacionadas à punção femoral durante o período peri-procedimento. Não houve elevação dos níveis séricos de creatinina $(0,96 \pm$ $0,29 \mathrm{mg} / \mathrm{dL}$ inicial vs. 0,93 $\pm 0,32 \mathrm{mg} / \mathrm{dL}$ aos 6 meses), denotando manutenção da taxa de filtração glomerular durante o seguimento (Figura 2).

\section{Segurança vascular renal}

Arteriografia renal foi realizada em todos os pacientes aos 6 meses de seguimento. Em um caso, houve evidência de estenose de artéria renal esquerda de grau significativo (70\%). Embora sem repercussão clínica, a mesma foi tratada com implante de stent, sem intercorrências.

\section{Efeitos sobre a pressão arterial e número de anti-hipertensivos aos 12 meses}

Após 12 meses de seguimento, a DSR reduziu a PA aferida em consultório, em média, 41,1 \pm 32,2/18,6 \pm

TABELA 2

Características angiográficas e técnicas do procedimento

\begin{tabular}{lc}
\hline Variável & $\mathbf{n = 9}$ \\
\hline Diâmetro da ARD/ARE, mm & $5,0 \pm 0,4 / 5,0 \pm 0,7$ \\
Extensão da ARD/ARE, mm & $33,7 \pm 7,0 / 26,4 \pm 10,3$ \\
Volume de contraste, $\mathrm{mL}$ & $76,7 \pm 25,4$ \\
Tempo de fluoroscopia, min & $19,7 \pm 6,2$ \\
Número de aplicações de radiofrequência & $5,2 \pm 0,7 / 4,3 \pm 1,7$ \\
na ARD/ARE & \\
\hline ARD: artéria renal direita; ARE: artéria renal esquerda. & \\
\hline
\end{tabular}

$15,2 \mathrm{mmHg}(p=0,04$ para PAS e $p=0,08$ para PAD) (Figura 3). Houve redução média de $2 \pm 2,3$ anti-hipertensivos prescritos aos 12 meses $(7,7 \pm 1,3$ inicial vs. 5,7 $\pm 2,2$ aos 12 meses, $p=0,03$ ) (Figura 4).

Observamos resposta anti-hipertensiva em oito (88,9\%) pacientes, com redução de até $104 \mathrm{mmHg}$ na PAS. Um único paciente não reduziu seus níveis pressóricos. Notamos também a redução de até seis fármacos anti-hipertensivos aos 12 meses de seguimento.

\section{DISCUSSÃO}

Este foi um estudo realizado em centro terciário de cardiologia, que utilizou cateter irrigado para aplicação de radiofrequência no contexto de DSR em hipertensos resistentes. Os resultados demonstraram que a ablação da atividade simpática renal utilizando cateter irrigado é factível, segura e eficaz.

O procedimento reduziu PAS e PAD aferidas em consultório, em média, 41,1 \pm 32,2 mmHg e 18,6 \pm $15,2 \mathrm{mmHg}$, respectivamente. Houve redução média de $2 \pm 2,3$ fármacos anti-hipertensivos. Notamos redução de até seis anti-hipertensivos e de até $104 \mathrm{mmHg}$ na PAS durante o seguimento. O Symplicity HTN-3 não reporta mudanças no número de anti-hipertensivos e a redução média na PAS foi de apenas $2,4 \mathrm{mmHg}$ quando comparada ao grupo controle.

A diminuição da PAS no HTN-3 foi observada tanto no grupo DSR como no grupo controle - um achado divergente dos estudos prévios; ademais, o decréscimo foi aproximadamente a metade daquele observado no Symplicity HTN-2, não obstante o fato de as pressões sanguíneas iniciais serem semelhantes nos dois estudos. Isso é intrigante, porque o grau de redução da PA está relacionado aos níveis tensionais pré-tratamento. Comparação entre os grupos controles dos estudos Symplicity HTN-3 e HTN-2 demonstra uma redução maior da PA no primeiro. É concebível que uma maior exposição à espironolactona $(28,7 \%$ vs. $17 \%$ dos pacientes) no Symplicity HTN-3 facilitou

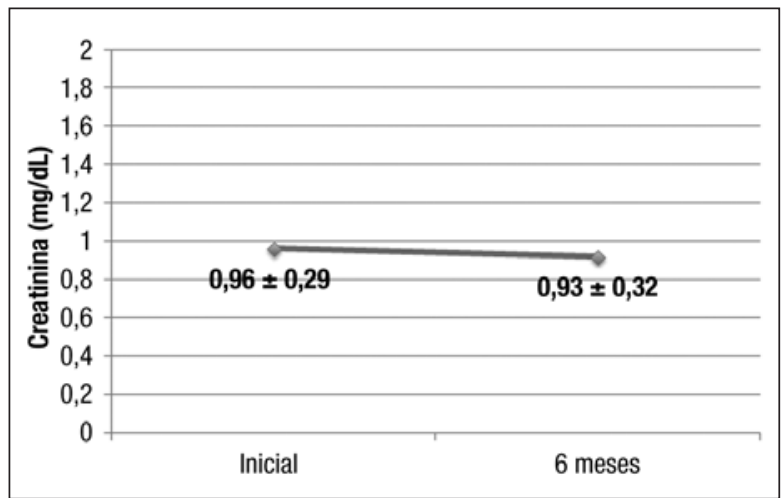

Figura 2. Efeitos da denervação simpática renal sobre a creatinina sérica nos primeiros 6 meses de evolução. 


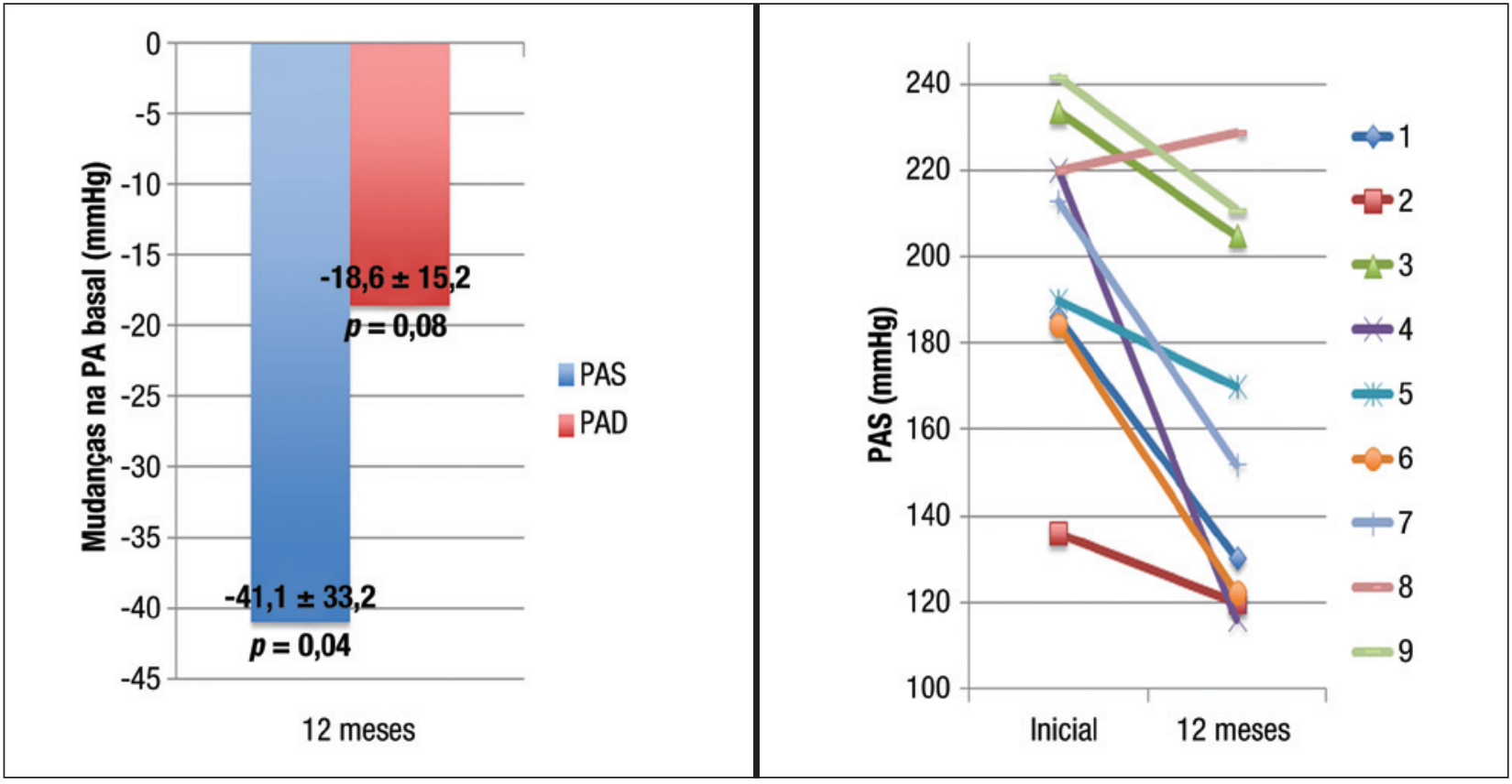

Figura 3. À esquerda, efeito da denervação simpática renal sobre a pressão arterial (PA) aferida em consultório. À direita, efeito da denervação simpática renal sobre a pressão arterial sistólica (PAS) aferida em consultório em cada um dos nove pacientes submetidos ao procedimento. PAD: pressão arterial diastólica.

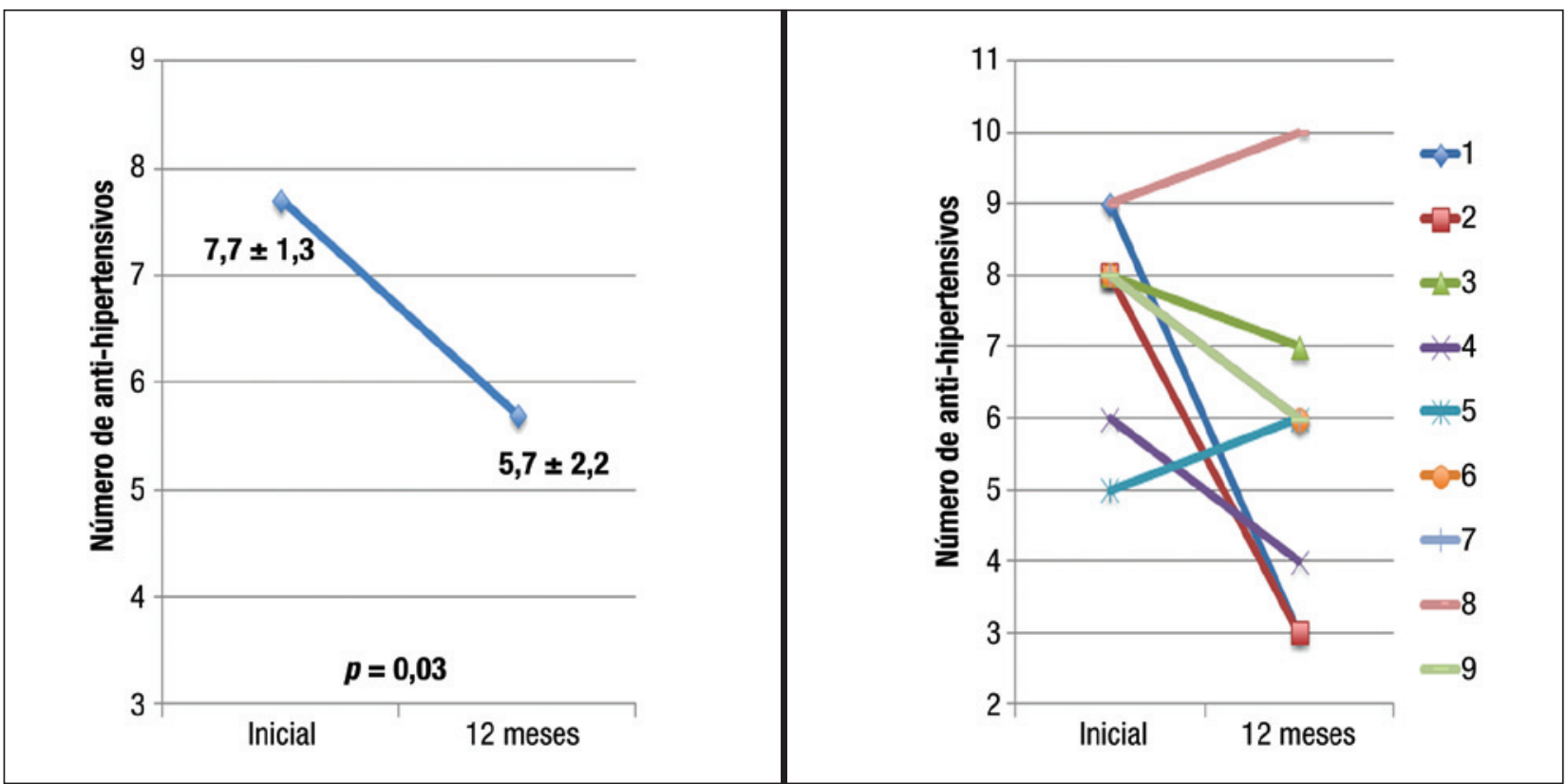

Figura 4. À esquerda, efeito da denervação simpática renal sobre o número de anti-hipertensivos. À direita, efeito da denervação simpática renal sobre o número de anti-hipertensivos em cada em cada um dos nove pacientes submetidos ao procedimento.

essa diminuição. Distintamente dos ensaios citados, a maioria dos pacientes da nossa população estava em uso de espironolactona $(88,9 \%)$.

A grande variabilidade na resposta à DSR levanta a questão de quanto esse procedimento poderia ser mais eficaz em pacientes selecionados, sobretudo naqueles com hiperatividade simpática. A taxa de não respondedores ao tratamento com DSR (definido como redução de PAS $<10 \mathrm{mmHg}, 6$ meses após o procedimento) varia de 8 e $37 \%$ nos principais estudos clínicos. As causas desse achado ainda não são completamente 
compreendidas, entretanto, algumas hipóteses podem ser formuladas com base no conhecimento da fisiopatologia da HAS e do procedimento de DSR. Dentre elas, enfatizamos: (1) a significativa variabilidade de dispositivos e técnicas, incluindo energia utilizada, número de eletrodos e duração da ablação; (2) a real relevância da hiperatividade simpática na fisiopatologia da hipertensão resistente, visto que esta é uma doença multifatorial e que, em alguns casos, a contribuição do sistema nervoso simpático pode não ser tão importante para a expressão da doença; (3) a seleção inapropriada de pacientes, incluindo portadores de pseudorresistência e HAS secundária; e (4) condições relacionadas ao paciente, como a não aderência ao tratamento farmacológico e a falha na mudança de estilo de vida. Os desvios padrão das mudanças na PAS inicial aferida em consultório, em ambos os grupos, DSR e controle, nos estudos Symplicity HTN-1 e HTN-2, foram amplos e muito semelhantes, indicando grande variação na resposta. A alteração na PAS em 95\% dos pacientes no Symplicity HTN-2 foi entre $-78 \mathrm{mmHg}$ e $14 \mathrm{mmHg}$, no grupo DSR, e entre $-43 \mathrm{mmHg}$ e $41 \mathrm{mmHg}$, no grupo controle. No Symplicity HTN-3, a média da redução da PA foi também dentro dessa faixa para os dois grupos. Na nossa população, o desvio padrão foi de $33,2 \mathrm{mmHg}$, e a alteração na PAS entre $-104 \mathrm{mmHg}$ e $9 \mathrm{mmHg}$. Não obtivemos resposta satisfatória em apenas um paciente $(11,1 \%)$.

Outras diferenças entre o Symplicity HTN-3 e nosso estudo merecem ser mencionadas. Primeiro, nossos pacientes necessitavam de maior número de fármacos anti-hipertensivos no início $(7,6 \pm 1,3$ vs. 5,1 $\pm 1,4)$. Segundo, em decorrência do menor número de pacientes, foi possível confirmar a HAS resistente com base na internação hospitalar, enquanto que o Symplicity HTN-3 definiu o diagnóstico em consultas ambulatoriais, fato que pode ter superestimado os níveis pressóricos basais. Terceiro, no Symplicity HTN-3, alterações nas doses dos fármacos anti-hipertensivos só eram permitidas caso o paciente apresentasse sintomas relacionados à hipotensão. No presente estudo, a dose dos fármacos foi alterada almejando o controle preconizado pela VI Diretriz Brasileira de Hipertensão Arterial; ${ }^{10}$ nos pacientes nos quais a PA basal estava controlada com quatro ou mais anti-hipertensivos, não houve redução significativa da PA e sim do número de fármacos no seguimento. Finalmente, utilizamos um cateter não dedicado para DSR - o cateter de ponta irrigada designado para ablação cardíaca. Acreditamos que seja esse o grande diferencial da nossa abordagem na DSR, permitindo-nos alcançar resultados superiores.

Publicamos os efeitos de três cateteres distintos, usualmente utilizados para ablação cardíaca, num experimento in vitro com artérias renais porcinas. ${ }^{11}$ Constatamos que o cateter irrigado (usualmente indicado para ablações de arritmias em câmaras cardíacas localizadas à esquerda) gera lesões mais profundas, causando destruição completa dos nervos, fato útil para promover a ablação daqueles situados além de $4 \mathrm{~mm}$ da camada íntima arterial, que podem compor até $40 \%$ do total de nervos nas artérias renais. Nossos resultados foram consistentes com os publicados por Ahmed et al., ${ }^{12}$ que descreveram dez pacientes com HAS resistente submetidos à DSR com cateter irrigado. Os autores demonstraram reduções na PAS e PAD de 21 e $11 \mathrm{mmHg}$, respectivamente, aos 6 meses de seguimento, e ausência de complicações graves.

Entusiasmo sobre DSR tem sido generalizado, como ilustrado nas declarações: "o potencial da denervação renal é enorme" e "pode ser usada não só para tratar a hipertensão, mas também doenças que caracterizam-se pela hiperatividade simpática, como diabetes e hiperinsulinemia, insuficiência cardíaca, arritmias e insuficiência renal crônica".$^{13}$ Essas palavras, cuidadosamente redigidas, citam os benefícios de denervação renal nessas alterações metabólicas ou cardiovasculares. Em contrapartida, as conclusões atuais do Symplicity HTN-3 por um dos mesmos autores, 6 meses mais tarde, atestam que "um efeito significativo sobre a PAS não foi observado. Avaliação adicional em ensaios clínicos rigorosamente delineados será necessária para confirmar os benefícios previamente relatados da DSR em pacientes com hipertensão resistente". A pergunta ainda sem resposta é qual o real papel da DSR como tratamento adjunto na HAS. A ciência exige cautela e o tempo responderá a esse questionamento.

\section{Limitações}

Dentre as principais limitações deste estudo, destacamos o desenho não randomizado, a pequena amostra e a ausência de um grupo controle. Apesar dessas limitações, os resultados parecem promissores e são concordantes com a literatura existente. Estudos randomizados e controlados com procedimento simulado são necessários para confirmar nossos resultados.

\section{CONCLUSÕES}

A denervação simpática renal percutânea utilizando o cateter de ablação por radiofrequência de ponta irrigada se mostrou factível, segura e eficaz.

\section{CONFLITO DE INTERESSES}

Não há.

\section{FONTE DE FINANCIAMENTO}

Não há.

\section{REFERÊNCIAS}

1. Schlaich MP, Sobotka PA, Krum H, Lambert E, Esler MD. Renal sympathetic-nerve ablation for uncontrolled hypertension. $N$ Engl J Med. 2009;361(9):932-4. 
2. Messerli FH, Bangalore S. Treatment-resistant hypertension: another Cinderella story. Eur Heart J. 2013;34(16):1175-7.

3. Krum H, Schlaich $M$, Whitbourn R, Sobotka PA, Sadowski J, Bartus $\mathrm{K}$, et al. Catheter-based renal sympathetic denervation for resistant hypertension: a multicentre safety and proofof-principle cohort study. Lancet. 2009;373(9671):1275-81.

4. Symplicity HTN-2 Investigatiors; Esler MD, Krum H, Sobotka PA, Schlaich MP, Schmieder RE, Böhm M. Renal sympathetic denervation in patients with treatment-resistant hypertension (The Symplicity HTN-2 Trial): a randomised controlled trial. Lancet. 2010;376(9756):1903-9.

5. Krum H, Schlaich MP, Sobotka PA, Böhm M, Mahfoud F, Rocha-Singh $\mathrm{K}$, et al. Percutaneous renal denervation in patients with treatment-resistant hypertension: final 3-year report of the symplicity HTN-1 study. Lancet. 2014;383(9917): 622-9.

6. Bhatt DL, Kandzari DE, O'Neill WW, D'Agostino R, Flack JM, Katzen BT, et al. A controlled trial of renal denervation for resistant hypertension. N Engl J Med. 2014;370(15):1393-401.

7. Fadl Elmula FE, Hoffmann P, Larstorp AC, Fossum E, Brekke $M$, Kjeldsen $S E$, et al. Adjusted drug treatment is superior to renal sympathetic denervation in patients with true treatmentresistant hypertension. Hypertension. 2014;63(5):991-9.
8. Armaganijan L, Staico R, Moraes A, Abizaid A, Moreira D, Amodeo $\mathrm{C}$, et al. Renal denervation using an irrigated catheter in patients with resistant hypertension: a promising strategy? Arq Bras Cardiol. 2014 Feb 17 [Epub ahead of print]

9. Calhoun DA, Jones D, Textor S, Goff DC, Murphy TP, Toto RD, et al. Resistant hypertension: diagnosis, evaluation, and treatment: a scientific statement from the American Heart Association Professional Education Committee of the Council for High Blood Pressure Research. Circulation. 2008;117(25):e510-26.

10. Sociedade Brasileira de Cardiologia; Sociedade Brasileira de Hipertensão, Sociedade Brasileira de Nefrologia. VI Diretrizes Brasileiras de Hipertensão. Arq Bras Cardiol. 2010; 95(1 Supl 1):1-51.

11. Staico R, Armaganijan L, Dietrich C, Abizaid A, Moreira D, Lopes $\mathrm{R}$, et al. Ablação da atividade simpática renal com cateter de ponta irrigada: uma opção atraente? Rev Bras Cardiol Invasiva. 2013;21(1):7-12.

12. Ahmed H, Neuzil P, Skoda J, Petru J, Sediva L, Schejbalova M, et al. Renal sympathetic denervation using an irrigated radiofrequency ablation catheter for the management of drug-resistant hypertension. JACC. Cardiovasc Interv. 2012;5(7):758-65.

13. Mahfoud F, Bhatt DL. Catheter-based renal denervation: the black box procedure. JACC Cardiovasc Interv. 2013;6(10):1092-4. 